\title{
Anlotinib-induced acute myocardial infarction: A case report and literature review
}

\author{
GUIHONG LIU ${ }^{1}$, TAO CHEN ${ }^{2}$ and ZHENYU DING $^{1}$ \\ ${ }^{1}$ Department of Biotherapy, Cancer Center, State Key Laboratory of Biotherapy, West \\ China Hospital, West China Medical School, Sichuan University; ${ }^{2}$ Cardiology Department, \\ Chengdu No. 7 People's Hospital, Chengdu Tumor Hospital, Chengdu, Sichuan 610041, P.R. China
}

Received September 12, 2019; Accepted May 29, 2020

DOI: $10.3892 / \mathrm{etm} .2020 .9041$

\begin{abstract}
Anlotinib hydrochloride is a multi-target tyrosine kinase inhibitor, which has been recently approved for the treatment of advanced non-small cell lung cancer in China. One of its mechanisms of action is the inhibition of angiogenesis and it is similar to other anti-angiogenesis drugs, as it has cardiovascular toxicity, which may damage vascular endothelial cells and result in hypertension and hyperlipidemia. All of the aforementioned factors are considered risk factors for coronary heart disease; however, the risk of developing acute myocardial infarction (AMI) has not been assessed by any previous clinical trials and subsequent research. The present case study, to the best of our knowledge, was the first to report on a patient who developed hypertension, hyperlipidemia and angina pectoris, and eventually experienced AMI, following treatment with anlotinib. This indicates that patients receiving anlotinib may require further observation and monitoring during use.
\end{abstract}

\section{Introduction}

Lung cancer is the most frequently diagnosed cancer worldwide, comprising $20 \%$ of all cancer-related mortalities (1). Non-small cell lung cancer (NSCLC) accounts for $80-85 \%$ of lung cancer cases (2). Anlotinib hydrochloride is a multi-target tyrosine kinase inhibitor, which is able to prolong progression-free survival and overall survival in patients with advanced NSCLC, who have an advanced stage of the disease despite receiving $>2$ types of systemic chemotherapy (3). Anlotinib is able to inhibit cancer progression and targets factors associated with angiogenesis, including

Correspondence to: Professor Zhenyu Ding, Department of Biotherapy, Cancer Center, State Key Laboratory of Biotherapy, West China Hospital, West China Medical School, Sichuan University, 37 Guoxue Lane, Chengdu, Sichuan 610041, P.R. China E-mail: dingzhenyu@scu.edu.cn

Key words: lung cancer, anlotinib hydrochloride, acute myocardial infarction vascular endothelial growth factor (VEGF) receptors 1-3, fibroblast growth factor (FGF) receptors 1-4, platelet-derived growth factor (PDGF) receptor $\alpha$ and $\beta$ and stem cell factor receptor (4). Various types of cardiovascular toxicity have been reported in the ALTER 0302 and 0303 clinical trials, including hypertension, sinus tachycardia and QTc prolongations; however, acute myocardial infarction (AMI) has not been reported $(3,5)$. The present case study, to the best of our knowledge, was the first to report on a case with advanced squamous cell carcinoma (SqCC) who developed AMI following treatment with anlotinib.

\section{Case report}

A 49-year-old male with a history of smoking (20 packs per year) and without coronary heart disease, hypertension, diabetes mellitus or hyperlipidemia presented with a cough and dyspnea in June 2015 at the West China Hospital (Chengdu, China). A CT scan revealed a shadow of the left lower lobe. A pathological diagnosis of T3N0M0 (stage II) $\mathrm{SqCC}$ was made following radical resection according to the eighth edition of the TNM classification of lung cancer (6). No epidermal growth factor receptor, ALK or ROS-1 mutations were identified. The patient refused adjuvant treatment following surgery and experienced back pain in March 2017. An additional CT scan indicated the third thoracic vertebral metastasis. The patient was subsequently prescribed five cycles of paclitaxel and cisplatin chemotherapy and $44 \mathrm{~Gy} / 22 \mathrm{f}$ radiotherapy and achieved partial response. The CT performed at reexamination in October 2017 revealed that the thoracic spine lesion was larger than before and was accompanied by left hilar lymph node enlargement. A total of 2 cycles of docetaxel and lobaplatin chemotherapy were subsequently administered to the patient and stable disease (SD) was achieved. To improve the efficacy, treatment was changed to 2 cycles of gemcitabine and cisplatin chemotherapy and SD was maintained. In April 2018, the CT scan revealed the tumor had invaded the spinal cord. The patient received $33 \mathrm{~Gy} / 30 \mathrm{f}$ radiotherapy for the metastatic masses prior to systemic treatment. In June 2018, the blood pressure, glucose and lipid levels of the patient were all within normal ranges (Table I); therefore, the patient was prescribed anlotinib (12 mg orally, once a day), until AMI was diagnosed (Fig. 1). 
After a month of using anlotinib, the patient developed occasional chest pain, high blood pressure $(140 / 100 \mathrm{mmHg})$, hyperlipidemia and hand-foot syndrome. The patient ignored the thoracalgia which had developed, resulting in more frequent and serious attacks requiring the use of nitroglycerin to relieve the pain, one week prior to AMI. On August 7, 2019, the patient presented with unbearable chest pain, which was not relieved by nitroglycerin. The electrocardiogram revealed atrial fibrillation, ST elevation in leads AVR and V1 (AVR>V1) and ST depression in leads V2 to V6, II, III and AVF (Fig. 2) with the amount of cardiac isoform of troponin $\mathrm{T}$ reaching $140.70 \mathrm{pg} / \mathrm{ml}$ (normal range, $0-14 \mathrm{pg} / \mathrm{ml}$ ). The cardiologists considered that the patient had experienced AMI. Emergency coronary angiography revealed $85 \%$ proximal stenosis of the left major coronary artery and coronary intervention with a drug-eluting stent was performed (Fig. 3). The patient did not experience any further chest pain and the postoperative electrocardiogram was normal (Fig. 4). Subsequently, the patient received dual antiplatelet therapy and stopped the use of anlotinib. The patient is still alive following the administration of regular immunotherapy.

\section{Discussion}

Coronary heart disease, hypertension, diabetes mellitus and hyperlipidemia, which are risk factors for AMI, were not previously observed in the middle-aged patient of the present study, who only exhibited a history of smoking prior to treatment with anlotinib (7). Several types of chemotherapeutic drugs have been administered following recurrence. The occurrence of cisplatin-associated AMI between the first dose and 4 weeks after the last dose has been reported (8). In several cases, the use of taxanes was reported to induce AMI $(9,10)$, and percutaneous coronary intervention with a paclitaxel-coated balloon is widely used to remedy this (11). Gemcitabine was only reported to cause peripheral ischemia and Raynaud's phenomenon in resistant arteries (12), while lobaplatin was not observed to cause any vascular lesions (13). Radiotherapy may cause damage to the vasculature and the rates of coronary events were demonstrated to increase linearly with increasing dosages directly exposed to the heart (14). AMI caused by radiotherapy is primarily observed in patients with breast cancer and Hodgkin's lymphoma due to the high dosages exposed to the heart (15). The incidence of AMI is relatively lower in patients with lung cancer due to the low cardiac dose and the improvements in radiation techniques, which avoid radiation exposure of the heart (16). In the present case, the radiotherapy site of the patient was located in the third thoracic spine region, indicating that the anatomic location of the lesion was higher than that of the heart. In other words, the heart of the patient was not as exposed as the location of lesion. The patient suffered angina pectoris following 1 month of treatment with anlotinib, indicating that pathological changes occurred in the coronary artery. Therefore, the AMI that had developed in the patient was reasonably attributed to anlotinib (Fig. 5).

Anlotinib serves an anti-tumor role by inhibiting several types of tyrosine kinases (4). In 1971, Folkman (17) proposed that tumor growth depends on angiogenesis and numerous anti-angiogenesis drugs have since emerged, including anlotinib. Vessels are lined by a single layer of endothelial cells
Table I. Laboratory parameters of the patient in June 2018.

\begin{tabular}{lcc}
\hline Parameter & Value & Normal range \\
\hline Blood pressure $(\mathrm{mmHg})$ & $128 / 60$ & $(90-139) /(60-89)$ \\
Fasting glucose $(\mathrm{mmol} / \mathrm{l})$ & 5.12 & $3.9-5.9$ \\
Cholesterol $(\mathrm{mmol} / \mathrm{l})$ & 4.29 & $2.8-5.7$ \\
Triglyceride $(\mathrm{mmol} / \mathrm{l})$ & 0.54 & $0.29-1.83$ \\
HDL-C $(\mathrm{mmol} / \mathrm{l})$ & 1.35 & $>0.9$ \\
LDL-C $(\mathrm{mmol} / \mathrm{l})$ & 2.83 & $<4.0$ \\
\hline
\end{tabular}

HDL-C, high-density lipoprotein cholesterol; LDL-C, low-density lipoprotein cholesterol.

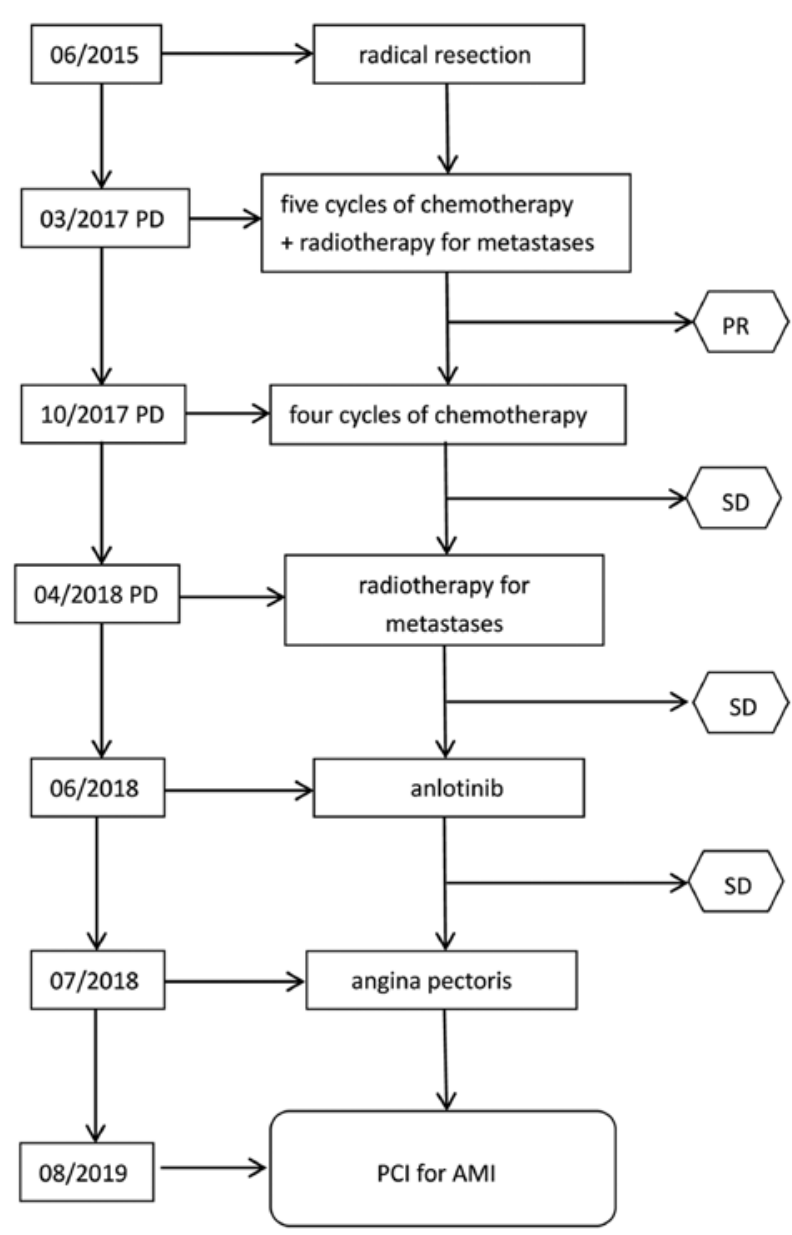

Figure 1. Flow chart of the treatment process. The patient initially received radical resection in June 2015 and was diagnosed with T3N0M0 (stage IIb) squamous cell carcinoma. Further treatment was administered following a CT scan indicating thoracic vertebral metastasis in March 2017, which subsequently resulted in the patient receiving anlotinib, and the development of AMI 14 months later. PD, progressive disease; PR, partial response; $\mathrm{SD}$, stable disease; CR, complete response; AMI, acute myocardial infarction; PCI, percutaneous coronary intervention.

that rely on VEGF and are surrounded by smooth muscle cells $(18,19)$. Arteries have a muscular layer that regulates the vascular tone, whereas capillaries are more sparsely supported by specialized mesenchymal cells known as pericytes, and depend on the production of PDGF by endothelial cells (20). Tumor cells secrete FGF to promote excess growth and 


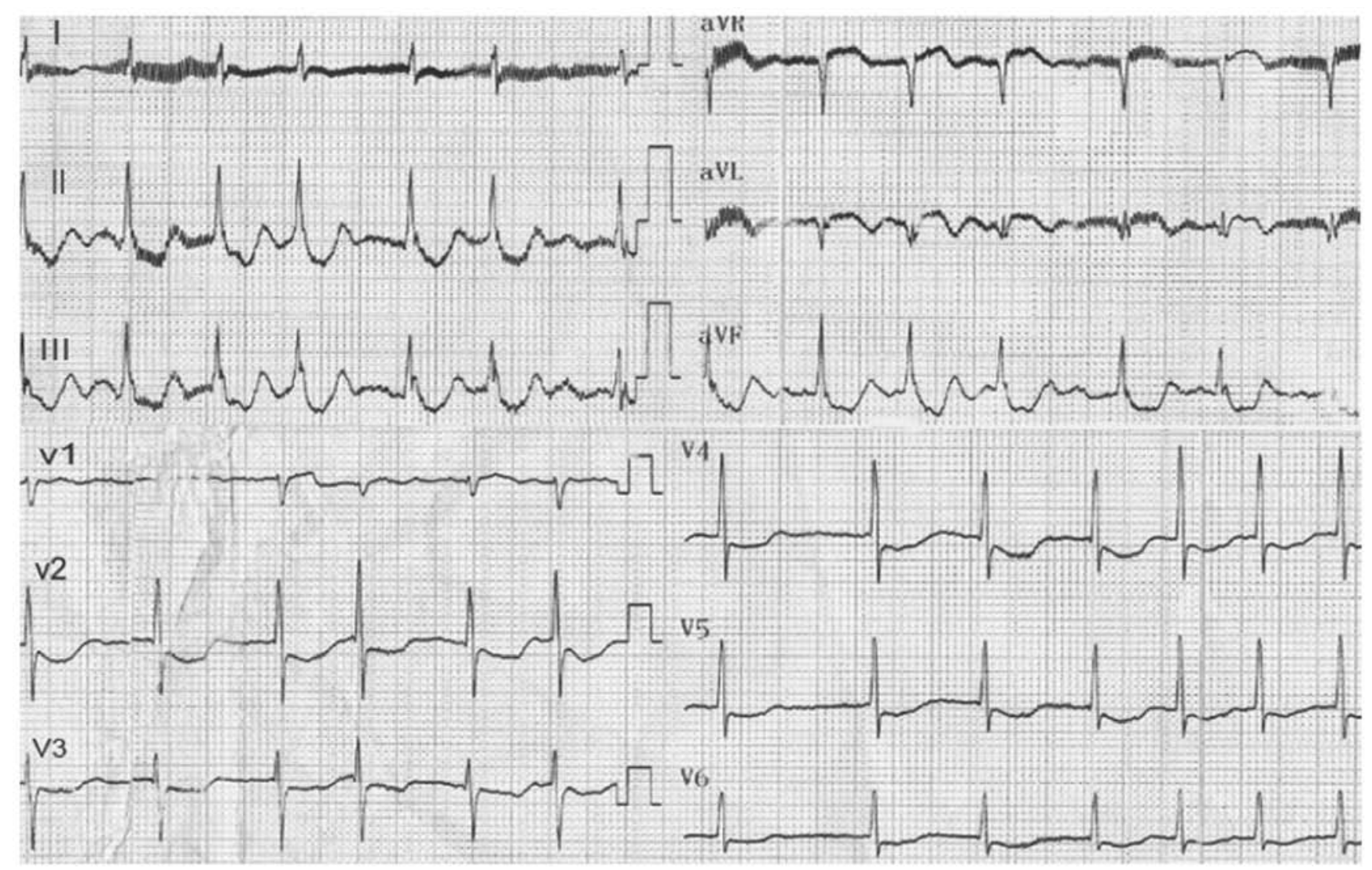

Figure 2. Initial electrocardiogram demonstrating atrial fibrillation, ST elevation in AVR and V1 (AVR>V1) and ST depression in V2 to V6, II, III and AVF.

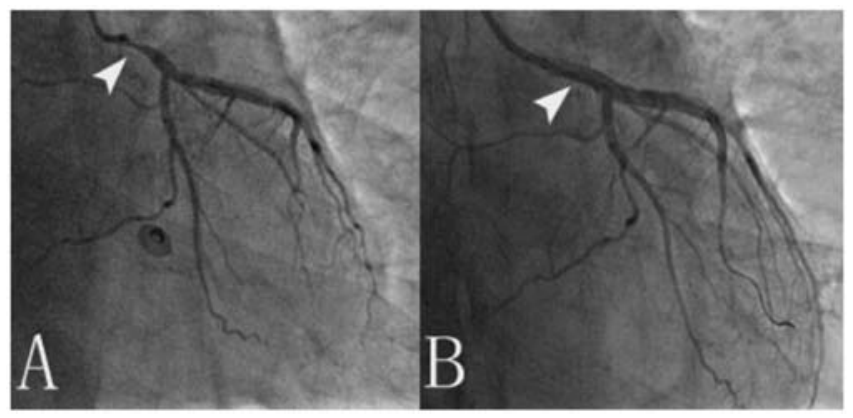

Figure 3. Coronary angiography. (A) Initial coronary angiography indicating $85 \%$ proximal stenosis of the left major coronary artery (arrowhead). (B) A drug-eluting stent was added to the left major coronary artery lesion $30 \mathrm{~min}$ subsequent to coronary angiography (arrowhead).

dysregulation of the vasculature (21). Anti-angiogenesis drugs exert effective tumor inhibition effects by suppressing the aforementioned factors (22).

Notably, toxicities also arise due to their mechanism. One such type is cardiovascular toxicity, including hypertension, congestive heart failure, cardiac ischemia and AMI (23). AMI is a fatal cardiovascular event, which is the result of cardiac ischemia (7). Coronary atherosclerosis and arterial thrombosis account for cardiac ischemia, and are formed due to the following factors: VEGF signaling pathway inhibitors increase the risk of arterial thrombosis, as they decrease endothelial cell survival and proliferation (24). Furthermore, administration of anti-angiogenesis drugs was hypothesized to decrease nitric oxide synthesis and lead to hypertension, which may increase endothelial damage (25). Hyperlipidemia is another side effect of anti-angiogenesis drugs, resulting in an acceleration of the formation of plaques and causing them to be unstable (7). A meta-analysis of angiogenesis inhibitors, including bevacizumab, aflibercept, ramucirumab, sunitinib, sorafenib, pazopanib, vandetanib, cabozantinib, axitinib, ponatinib and regorafenib, indicated that the risk of cardiac ischemia almost tripled and fatal cardiovascular events increased by 1.26-fold (23). Myocardial infarction has also been reported in association with the use of bevacizumab, ramucirumab, sunitinib and sorafenib (25-27).

Cardiovascular toxicity was notable in phase I, II and III clinical trials of anlotinib (3-5); however, to the best of our knowledge, the present study was the first to report on a case of anlotinib-induced AMI. The number of patients and follow-up time were limited in these clinical trials and the longest median duration of anlotinib treatment was 24 weeks in these clinical trials, a longer time period may be required to observe the occurrence of AMI in a larger population. Furthermore, anlotinib was only approved for the treatment of patients with advanced NSCLC who have progressed following treatment with $>2$ types of prior systemic chemotherapy (28). Thus, the effects of anlotinib are difficult to observe in a clinical setting, as the health of the patients is generally poor, with a short life expectancy (3-5). However, the patient in the present case study received anlotinib for $>1$ year, with a good response, which enabled the observation of the occurrence of AMI.

In conclusion, to the best of our knowledge, the present study was the first to report on a case of AMI induced by anlotinib. 


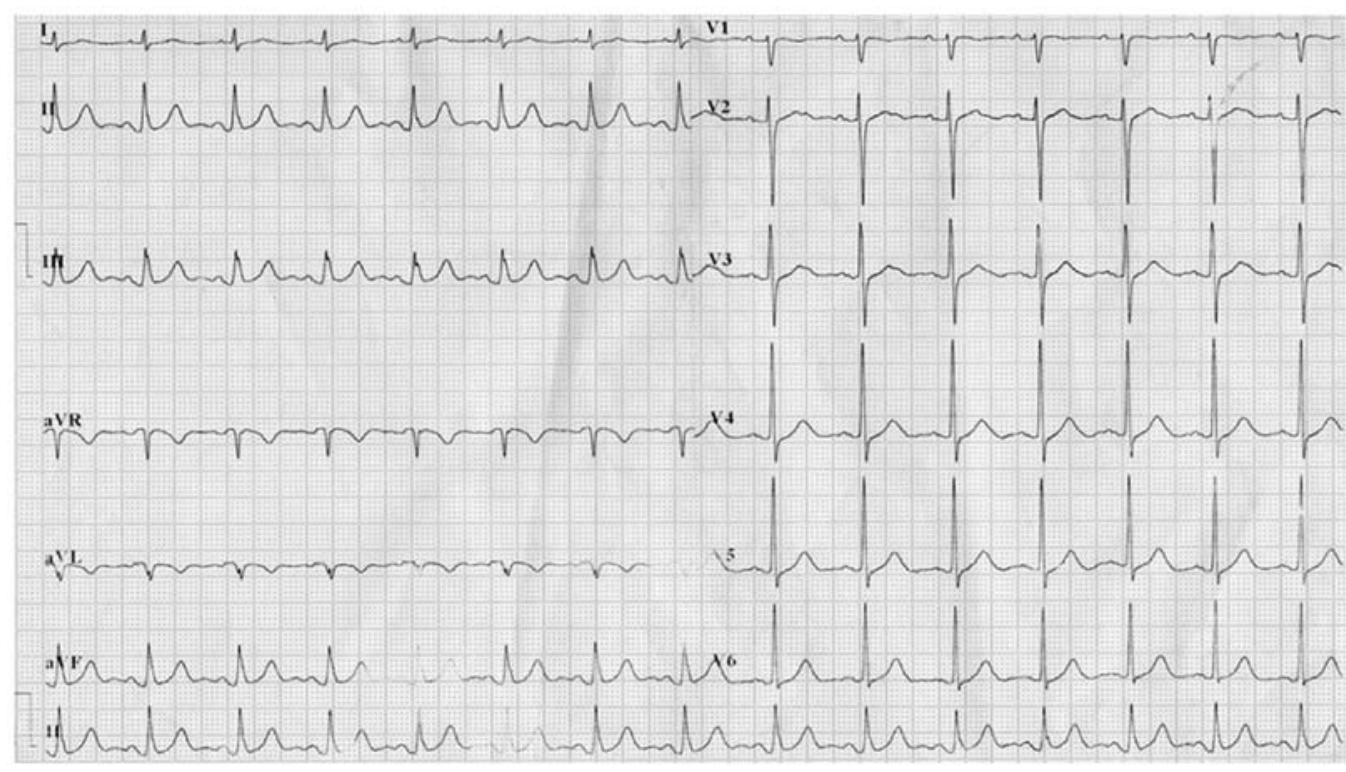

Figure 4. Electrocardiogram after half an hour of percutaneous coronary intervention demonstrating sinus rhythm and all abnormal leads were back to normal.

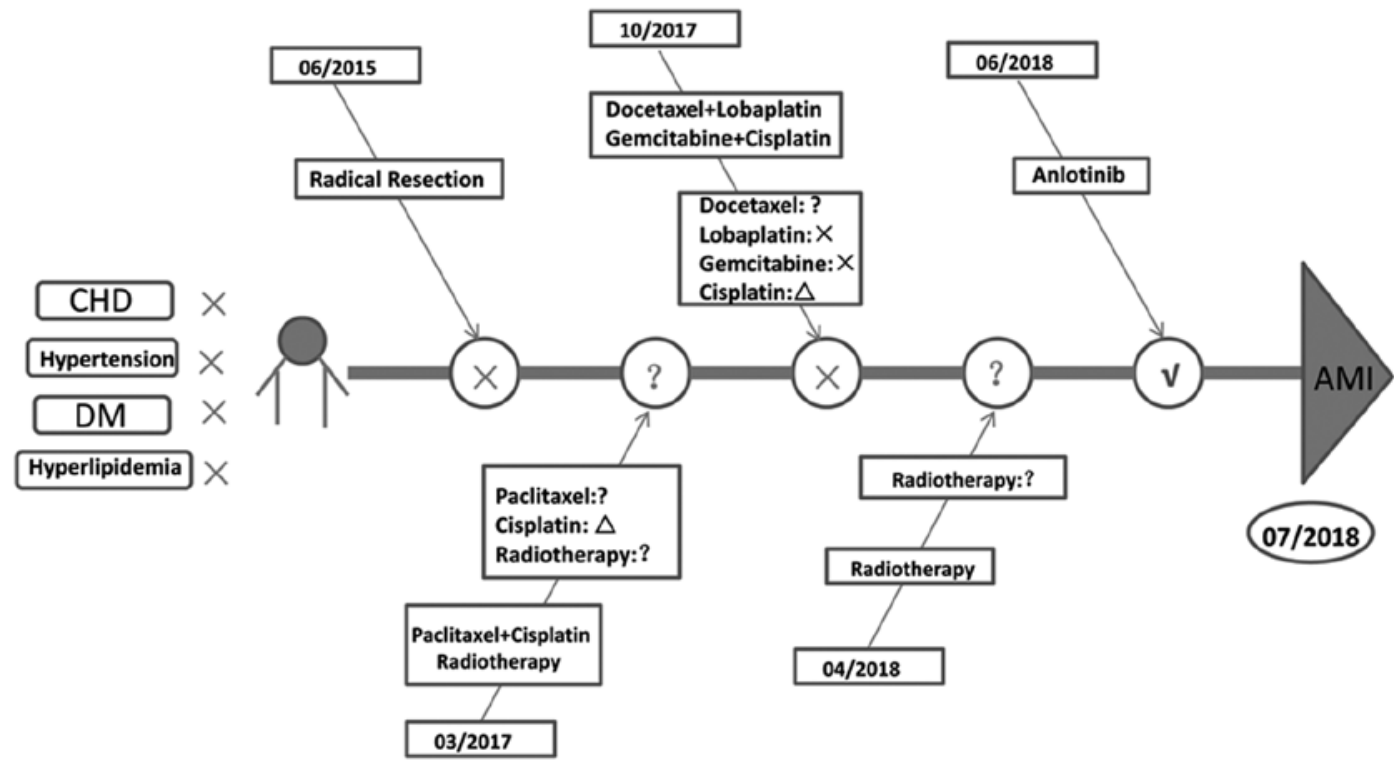

Figure 5. Analytical process of identifying the cause of AMI by anlotinib. CHD, hypertension, diabetes DM and hyperlipidemia were not exhibited by the patient. The patient received surgery, chemotherapy, radiotherapy and targeted drug therapy with anlotinib for the treatment of squamous cell carcinoma. The possibility of AMI following surgery, chemotherapy and radiotherapy was excluded. AMI was therefore attributed to anlotinib. ' $x$ ' indicates that the patient did not develop the disease/disorder or did not receive the type of treatment. ' $\Delta$ ' indicates that treatment with cisplatin was not able to induce AMI due to this effect being beyond its drug action cycle. '?' indicates the probabitiy of taxanes and radiotherapy causing AMI, which was uncertain. ' $\sqrt{ }$ ' indicated that this caused AMI. CHD, coronary heart disease; DM, diabetes mellitus; AMI, acute myocardial infarction.

Fatal cardiovascular events may also occur due to anlotinib, which is similar to the effects of other anti-angiogenesis drugs; therefore, patients treated with anlotinib require further and regular monitoring in the future.

\section{Acknowledgements}

Not applicable.

\section{Funding}

No funding was received.

\section{Availability of data and materials}

The datasets used and/or analyzed during the present study are available from the corresponding author on reasonable request.

\section{Authors' contributions}

GL analyzed and interpreted the data, and was a major contributor in drafting the manuscript. TC collected the clinical data and was involved in drafting the manuscript. ZD supervised the collection and interpretation of data and critically revised 
the manuscript. All authors read and approved the final version of the manuscript.

\section{Ethics approval and consent to participate}

The present study was approved by the Ethics Committee of Chengdu No. 7 People's Hospital, Chengdu Tumor Hospital, (Chengdu, China) and informed consent was obtained from the patient.

\section{Patient consent for publication}

The patient provided written informed consent regarding the publication of the case details and any associated images.

\section{Competing interests}

The authors declare that they have no competing interests.

\section{References}

1. Ferlay J, Soerjomataram I, Dikshit R, Eser S, Mathers C, Rebelo M, Parkin DM, Forman D and Bray F: Cancer incidence and mortality worldwide: Sources, methods and major patterns in GLOBOCAN 2012. Int J Cancer 136: E359-E386, 2015.

2. Miller KD, Siegel RL, Lin CC, Mariotto AB, Kramer JL, Rowland JH, Stein KD, Alteri R and Jemal A: Cancer treatment and survivorship statistics, 2016. CA Cancer J Clin 66: 271-289, 2016.

3. Han B, Li K, Wang Q, Zhang L, Shi J, Wang Z, Cheng Y, He J, Shi Y, Zhao Y, et al: Effect of anlotinib as a third-line or further treatment on overall survival of patients with advanced non-small cell lung cancer: The ALTER 0303 phase 3 randomized clinical trial. JAMA Oncol 4: 1569-1575, 2018.

4. Sun Y, Niu W, Du F, Du C, Li S, Wang J, Li L, Wang F, Hao Y, Li C and Chi Y: Safety, pharmacokinetics, and antitumor properties of anlotinib, an oral multi-target tyrosine kinase inhibitor, in patients with advanced refractory solid tumors. J Hematol Oncol 9: 105, 2016.

5. Han B, Li K, Zhao Y, Li B, Cheng Y, Zhou J, Lu Y, Shi Y, Wang Z, Jiang L, et al: Anlotinib as a third-line therapy in patients with refractory advanced non-small-cell lung cancer: A multicentre, randomised phase II trial (ALTER0302). Br J Cancer 118 654-661, 2018

6. Rami-Porta R, Bolejaek V, Giroux DJ, Chansky K, Crowley J, Asamura $\mathrm{H}$ and Goldstraw P; International Association for the Study of Lung Cancer Staging and Prognostic Factors Committee, Advisory Board Members and Participating Institutions: The IASLC lung cancer staging project: The new database to inform the eighth edition of the TNM classification of lung cancer. J Thorac Oncol 9: 1618-1624, 2014.

7. Anderson JL and Morrow DA: Acute myocardial infarction. N Engl J Med 376: 2053-2064, 2017.

8. Moore RA, Adel N, Riedel E, Bhutani M, Feldman DR, Tabbara NE, Soff G, Parameswaran R and Hassoun H: High incidence of thromboembolic events in patients treated with cisplatin-based chemotherapy: A large retrospective analysis. J Clin Oncol 29: 3466-3473, 2011.

9. Jordan MA and Wilson L: Microtubules as a target for anticancer drugs. Nat Rev Cancer 4: 253-265, 2004.

10. Rowinsky EK, McGuire WP, Guarnieri T, Fisherman JS, Christian MC and Donehower RC: Cardiac disturbances during the administration of taxol. J Clin Oncol 9: 1704-1712, 1991.

11. Ali RM, Abdul Kader MASK, Wan Ahmad WA, Ong TK, Liew HB, Omar AF, Zuhdi ASM, Nuruddin AA, Schnorr B and Scheller B: Treatment of coronary drug-eluting stent restenosis by a sirolimus- or paclitaxel-coated balloon. JACC Cardiovasc Interv 12: 558-566, 2019.
12. Dasanu CA: Gemcitabine: Vascular toxicity and prothrombotic potential. Expert Opin Drug Saf 7: 703-716, 2008.

13. Gu L, Zhong D, Yu T, Tang P, Meng F and Qin Q: Retrospective study of the efficacy and toxicity of lobaplatin-etoposide chemotherapy in small cell lung cancer. Thorac Cancer 10: 226-233, 2019.

14. Darby SC, Ewertz M, McGale P, Bennet AM, Blom-Goldman U, Brønnum D, Correa C, Cutter D, Gagliardi G, Gigante B, et al: Risk of ischemic heart disease in women after radiotherapy for breast cancer. N Engl J Med 368: 987-998, 2013.

15. Zamorano JL, Lancellotti P, Rodriguez Muñoz D, Aboyans V, Asteggiano R, Galderisi M, Habib G, Lenihan DJ, Lip GYH, Lyon AR, et al: 2016 ESC position paper on cancer treatments and cardiovascular toxicity developed underthe auspices of the ESC committee for practice guidelines: The task force for cancertreatments and cardiovascular toxicity of the European society of cardiology (ESC). Eur Heart J 37: 2768-2801, 2016.

16. Lee CC, Zheng H, Soon YY, Foo LL, Koh WY, Leong CN, Vellayappan B, Tey JCS and Tham IWK: Association between radiation heart dosimetric parameters, myocardial infarct and overall survival in stage 3 non-small cell lung cancer treated with definitive thoracic radiotherapy. Lung Cancer 120: 54-59, 2018.

17. Folkman J: Tumor angiogenesis: Therapeutic implications. N Engl J Med 285: 1182-1186, 1971.

18. Folkman J: Angiogenesis in cancer, vascular, rheumatoid and other disease. Nat Med 1: 27-31, 1995.

19. Ferrara N, Gerber HP and LeCouter J: The biology of VEGF and its receptors. Nat Med 9: 669-676, 2003.

20. Baluk P, Hashizume $\mathrm{H}$ and McDonald DM: Cellular abnormalities of blood vessels as targets in cancer. Curr Opin Genet Dev 15: 102-111, 2005.

21. Claesson-Welsh L: Blood vessels as targets in tumor therapy. Ups J Med Sci 117: 178-186, 2012.

22. Lugano R, Ramachandran M and Dimberg A: Tumor angiogenesis: Causes, consequences, challenges and opportunities. Cell Mol Life Sci 77: 1745-1770, 2020.

23. Abdel-Qadir H, Ethier JL, Lee DS, Thavendiranathan P and Amir E: Cardiovascular toxicity of angiogenesis inhibitors in treatment of malignancy: A systematic review and meta-analysis. Cancer Treat Rev 53: 120-127, 2017.

24. Ivy SP, Wick JY and Kaufman BM: An overview of small-molecule inhibitors of VEGFR signaling. Nat Rev Clin Oncol 6: 569-579, 2009.

25. Choueiri TK, Schutz FA, Je Y, Rosenberg JE and Bellmunt J: Risk of arterial thromboembolic events with sunitinib and sorafenib: A systematic review and meta-analysis of clinical trials. J Clin Oncol 28: 2280-2285, 2010.

26. Dahlberg SE, Sandler AB, Brahmer JR, Schiller JH and Johnson DH: Clinical course of advanced non-small-cell lung cancer patients experiencing hypertension during treatment with bevacizumab in combination with carboplatin and paclitaxel on ECOG 4599. J Clin Oncol 28: 949-954, 2010.

27. Herbst RS, Arkenau HT, Santana-Davila R, Calvo E, Paz-Ares L, Cassier PA, Bendell J, Penel N, Krebs MG, Martin-Liberal J, et al: Ramucirumab plus pembrolizumab in patients with previously treated advanced non-small-cell lung cancer, gastro-oesophageal cancer, or urothelial carcinomas (JVDF): A multicohort, non-randomised, open-label, phase 1a/b trial. Lancet Oncol 20: 1109-1123, 2019.

28. Zhou M, Chen X, Zhang H, Xia L, Tong X, Zou L, Hao R, Pan J, Zhao X, Chen D, et al: China national medical products administration approval summary: Anlotinib for the treatment of advanced non-small cell lung cancer after two lines of chemotherapy. Cancer Commun (Lond) 39: 36, 2019.

This work is licensed under a Creative Commons Attribution-NonCommercial-NoDerivatives 4.0 International (CC BY-NC-ND 4.0) License. 